\title{
Experimental and Computational Method for Determining Parameters of Stress-Strain State from the Data Obtainable by Interference Optical Techniques
}

\author{
A. Chernyatin ${ }^{1}$, I. Razumovsky ${ }^{2}$ \\ ${ }^{1}$ Applied Mechanics Department, Bauman Moscow State Technical University, Russia \\ ${ }^{2}$ Mechanical Engineering Research Institute, Russian Academy of Sciences, Russia
}

\begin{abstract}
Experimental and computational method for determining parameters of stress-strain state is proposed which is based on estimation of compliance between the data sets obtained experimentally and the results of numerical calculations of the boundary problems in formulation of which all distinctive features of area geometry, character of the loads being considered and deformation characteristics of materials are taken into account. The procedure proposed was checked at a number of practically important problems.
\end{abstract}

The methods for investigation into stress-strain state (SSS) of structures based on interference optical techniques for registration of strain or displacement fields (photoelastic techniques, holographic interferometry, speckle photography, electronic digital speckle pattern interferometry techniques) provide the acquisition of uninterrupted information at the surface of the object being studied $[1,2]$. These methods being commonly used to solve the problems of mechanics of deformed solids, fracture mechanics, vibration-based diagnostics, structural dynamics etc., have distinct advantages over traditional measuring tools that allows only averaged (accumulated over known time base) values of strains or displacements in one direction and at the point given to be registered. The adequacy of the results of such measurements requires, as a rule, additional substantiation.

In modern practice the photoelastic coatings technique and, in recent years, electronic digital speckle pattern interferometry (EDSI) became the most common among these methods; significant advantage of these techniques is the capability of creating compact and reliable measurement instrumentation which can be used effectively under full-scale conditions. Besides, the evolvement of modern computer technologies offers a considerable increase in accuracy of measurement results as well as development of conceptually new methodological approaches to solving mechanics problems.

The determination of SSS parameters for the object being studied on the basis of processing of the experimental data obtainable by interference optical techniques includes three main stages:

- registration of baseline information on the basis of one or another effect of interference optics (photoregistration of interference patterns, hologram recording, registration of images with speckle pattern etc.);

- presentation of obtainable information on electronic media in digital format in terms of phase differences of light waves or (after primary processing) directly through components of threedimensional vector of body surface point displacements;

This is an Open Access article distributed under the terms of the Creative Commons Attribution-Noncommercial License 3.0, which permits unrestricted use, distribution, and reproduction in any noncommercial medium, provided the original work is properly cited. 
- determination of SSS parameters on the basis of initial experimental information processing.

The SSS parameters to be determined may be both local characteristics - strains or stresses definable on the basis of processing of displacement fields, and integral characteristics - e.g., parameters of fracture mechanics (stress intensity factors - SIF, J-integral and others) or the values of internal power factors in cross-sections of the object being studied. The characteristics of materials deformation for determining which the methods based on processing of the interference patterns obtainable under specimen loading have been developed can also be assigned to integral characteristics [3 and others].

It is arguable that conventional methods for determining SSS parameters are based on common methodological approach based on the known correlations between desired parameters and experimental data underlie a priori. It is subject to both "the simplest" problems of determining strains according to displacement fields on the basis of Cauchy's relations, and more challenging problems. In the first place the following problems should be assigned to the last mentioned tasks:

- methods for determination of SIF or $J$-integrals with allowance for asymptotic solutions of canonical singular boundary problems for the bodies with cracks [2,4,5 and others];

- methods for determination if residual stresses (RS) with allowance for the known a priori models of their distribution in the object area being considered [1,4,6 and others].

In the present work the experimental and computational method for determination of SSS parameters that is based on evaluation of conformance between the data sets obtained through experiments and the results of calculations of the model boundary problems by numerical procedures; under setting up these boundary problems all peculiarities of area geometry, character of the loads being considered and material deformation characteristics are taken into account.

The procedure proposed for determination of SSS parameters is as follows. The desired SSS parameters $P_{j}$ (in capacity of which the values of forces, RS, SIF etc. may be considered) are determined on the basis of minimization of the objective function characterizing deviations of experimentally definable $e_{i}{ }^{*}$ parameters (both strains, and components of displacement vector etc. can be such parameters) at $N$ points from their calculation $e_{i}$ values, obtainable as a result of numerical solving direct problem at running $P_{j}$ values ( $N$ points of registration of experimental data at the surface of the area being considered). The appropriate procedure reduces to organization of iteration process, at each step of which the values of $P_{j}$ parameters and the $e_{i}$ values corresponding to them, and the $P_{j}$ values under which the objective function reaches the minimum level are taken as a solution. It should be noted that the problem involves determining just the values of $P_{j}$ parameters on the basis of the model of their distribution assumed in advance.

To solve the direct problem that consists, generally, in acquisition of response field and, in particular, in acquisition of values of the responses at given measurement points the FEM ANSYS bundled software is used in which specialized APDL-based macroses were developed to provide:

- parametric simulation of the area being considered with various peculiarities (e.g., material lamination is taken into consideration, holes and cracks may be included, etc.) and its automatic partition into elements - reasonable from viewpoint of accuracy and resource intensity;

- automatic formation of the holes in the area being considered the number and variants of mutual disposition of which are unlimited;

- automatic construction of spatial, arbitrarily oriented, flat cracks with arbitrarily prescribed geometry of front and simply ordered grid of singular elements around it at given area (the crack may be surface, internal or through, crack front may pass through unlimited number of volumes);

- automatic calculation of fracture mechanics parameters including their distribution along cfack front;

- introduction of desired $P_{j}$ problem parameters;

- formation of deformational response fields found on the basis of calculations and determination of $e_{i}$ values at measurement points. 
The emphasis was on development of the algorithms for construction of listed objects with realization of common generalized properties in them and on issues of construction of reasonable finite element grid.

It should be noted that in case of linear dependence of parameters $e_{i}$ and $P_{j}$ there is no need to solve the direct problem at each stage of objective function minimization. To accelerate calculation procedure at knowingly given points - points of measurements the compliance factors of the system from action of $P_{j}=1, P_{k \sim j}=0$ can be determined (in ANSYS environment). Then the vectors of response components - a kind of discrete response bank (RB) (in MATLAB environment) are created. On the principle of superposition and proportionality of the responses to loads (through compliances) the values of responses at arbitrary values of $P_{j}$ parameters can be calculated at all times. In the presence of nonlinearity of parameters it is possible to proceed as follows. The values $P_{j}$ are given with the help of the grid method. For various combinations of $P_{j}$ the responses are computed (in ANSYS environment), thereby for each measurement point response values is formed, on the basis of which the spline surface in hyperspace is "constructed" according to parameters. That is to say, the continual bank of responses is created collectionwise, and at arbitrary values of $P_{j}$ parameters the response can be computed at any considered point by interpolating calculated data with the help of appropriate spline surface (in MATLAB environment).

The procedure for search of objective function minimum is based on deforming simplex element method (Nelder-Mead method) realized in MATLAB software package. It is the method of unconstrained optimization having high robustness even at ill conditio sine qua non of problem [7]. Root-mean square or maximal departures of calculation displacement values from experimental data are taken as the objective function.

This methodology for determining SSS parameters refers to the class of inverse problems under developing the procedure of their solving there is a need to consider the issues of existence, uniqueness and stability (of solution). Choice of adequate model of the problem is a necessary criterion of problem solving.

During determination of deformation response parameters the experimental error takes place in any way. Therefore, in the course of investigation the series of numerical experiments is carried out with the aim to analyze solution stability under action of different factors. Appropriate procedure is as follows: direct check problem is solved at given values of desired parameters (e.g., loads over a distance from zone of measurements), and on the basis of results of this solution the values of the quantities defined experimentally (e.g. displacements at $N$ points placed at the lines of equal displacements corresponding to the interference patterns defined experimentally) are determined. Then the error generated in random manner for each point and not exceeding the given $\delta$ value corresponding to experimental error is introduced in these values. This particular data is considered as initial information for solving inverse problem according to described above (proposed numerical) methodology. Eventually the $P_{j}$ mathematical expectation and dispersion are determined (Figure 1).

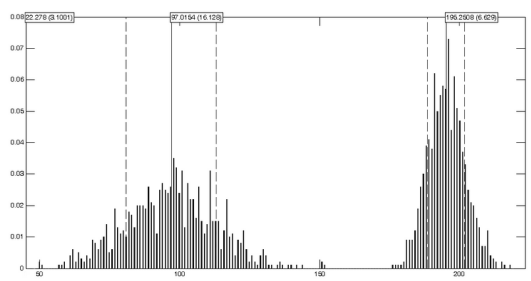

Fig. 1. Scattering histograms of desired parameters

The methodology for determining SSS parameters on the basis of the displacement fields (using EDSI technique) or strains (using photoelastic coating technique) data sets obtained experimentally is realized in the form of unified bundled software based on application of ANSYS and MATLAB software packages in their automatic interaction (Figure 2). It consists in: 


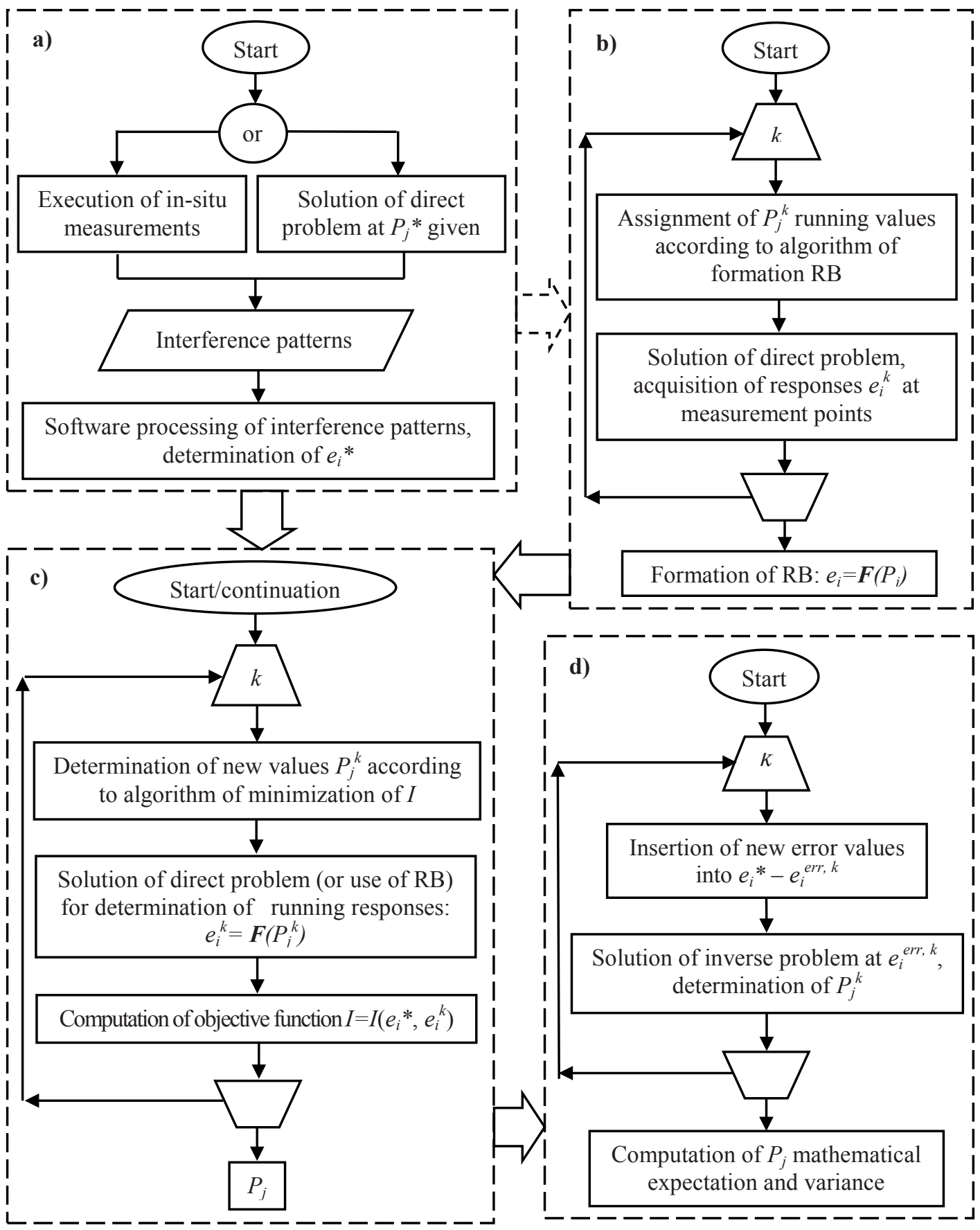

Fig. 2. Principal flowgraph for determination of SSS parameters on the basis of mathematical processing of experimental data:

a) processing of data sets (experiment or calculation); b) formation of response bank;

c) solution of inverse problem; d) stability analysis of solution 
- a program for processing of interference patterns (developed in MATLAB software environment) allowing to form the data sets of experiments on-line at arbitrary measurement points and all required information for further automatic application directly at the interference patterns downloaded to computer;

- a program (MATLAB) intend for realize direct solution of appropriate boundary problem at the geometry given and values of desired $P_{j}$ parameters and for create a kind of bank of $e_{i}$ responses;

- the main program (MATLAB) allowing to realize solution of inverse problem (in this particular case to fulfill computation of $P_{j}$ values on the basis of experimental $e_{i}{ }^{*}$ data and perform the analysis of influence of different factors (errors in data of experiments, geometry of area, initial estimates of desired $P_{j}$ parameters etc.) on solution stability;

- auxiliary macroses for different purposes realized in ANSYS environment;

- a number of subprograms of auxiliary character.

All programs in MATLAB software environment have graphic interface.

Solution of boundary problems of deformed solid mechanics in one software package (ANSYS) and realization of different mathematical algorithms (objective function minimization, automatic formation and use of response bank, data operations and others) in other software package (MATLAB) was made possible by development of special algorithm of automatic continuous data exchange between these software packages. Two programs were developed for realization, and each of them is described in its environment. To provide confunction of all programs and macroses destined for fulfillment of various functions under "auspices" of master program has been gained at the expense of using structured lists of parameters and data designated further as "protocols":

- protocol of model contains the parameters required for computer-aided finite-element modeling with all peculiarities, etc.;

- protocol of loading contains the data about distribution, magnitudes and cross correlation of parameters, etc.;

- protocol of materials contains data about mechanical characteristics of materials;

- protocol of measurements is created automatically by the program of image processing, contains information about patterns, positions of measurement points, magnitudes of responses at them and about "adaptation" of all data for FEM-model;

- protocol of problem solving contains sequence of $P_{j}$ parameters determination, calculation parameters and information about which data is needed to be used at each stage.

Application of the protocols allowed problem solving to be computerized in full. Any combination of parameters from these protocols can be used as search parameters.

Use of response bank allowed the time for each set up of numerical experiment to be shortened essentially.

The methodology proposed was tested for a number of problems. All problems had common setting up: the area of structure is considered in the form of prismatic body, along (side) boundaries of which (in two directions) the normal stresses act with definite law of distribution in depth. One hole or several holes are drilled at surface what leads to stress release and occurrence of deformation response fields at surface of the area. Practical problem lies in the fact that according to these fields there is a need to determine the values of stresses $\left(P_{j}\right.$ parameters) forming assumed model of distribution. The following problems had been considered and successfully solved. Application of EDSI technique or photoelastic coatings technique is foreseen as the methods for acquisition of initial experimental information.

1. Plate with stresses linearly varying in depth (membrane forces and bending moments). The fields of displacements in two (three) directions defined by EDSI technique were taken as deformation field. The investigation into influence of root-mean-square and maximal departure on solution convergence had been carried out, and high effectiveness of methodology application had been shown.

2. Half-space with surface elliptic crack and uniform fields of stresses (Figure $3 \mathrm{a}, \mathrm{b}$ ). Small holes are drilled in crack tips, thereby crack extension discontinues. Loading of given area can be 
estimated according to the fields of deformation response caused by hole formation [8]. The investigation into influence of the following factors on magnitudes of desired stresses (loads) had been carried out: scattering of the values of the displacements defined experimentally (with the help of EDSI technique or photoelastic coatings technique) conditioned by hole drilling in loaded structure; number and localization of measurement points. The necessary conditions for processing (preparation for solving) of experimental information had been formulated on the basis of obtained results. It is possible to define the characteristic sizes of the crack (for example, crack depth, including geometry of a crack set) together with loadings.

3. Problem of RS distribution in depth of bimetal reactor vessel sidewall. The piecewise linear stress distribution with stepwise change of residual stresses at the boundary of jump joint between dissimilar materials (Figure 3c) was considered [6]. As numerical experiment had shown, if determination of objective function is performed on the basis of the principles considered above then in that case considerable scattering of desired RS values takes place. In connection with this the special objective function was considered whose application allowed the RS values and distribution to be obtained with the accuracy sufficient for practical implementation.

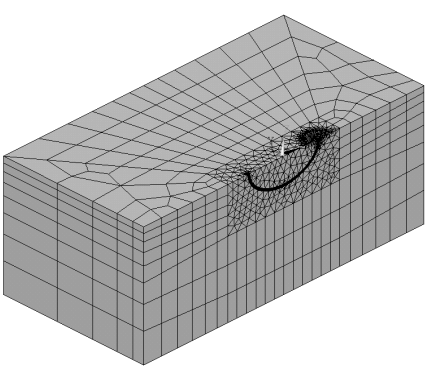

a)

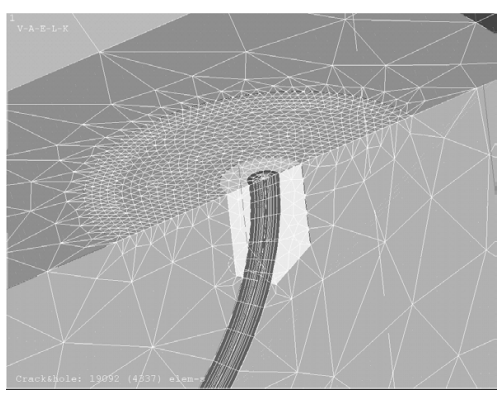

b)

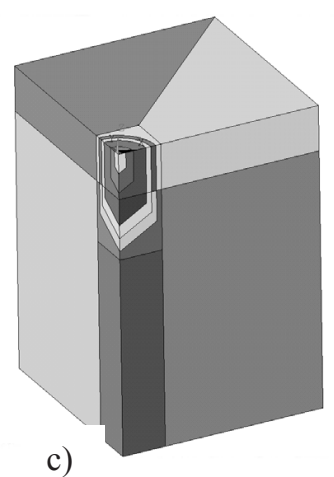

c)

Fig. 3. a) Finite elements model of half-space with surface elliptic crack; b) mesh of finite elements near the crack tip; c) structural model of half-space with dissimilar materials

Thus, the methodology proposed has been realized in the form of integrated software product based on continuous interaction between ANSYS and MATLAB software packages on the course of inverse problem solving. The system of developed (main and auxiliary) programs allows all stages of problem solving to be automatized: from indicating the localization places for selected points at deformation response fields, values of their displacements (obtained by EDSI technique) to checking correctness of design model definition and adequacy of the results obtainable in the course of problem solving (on the basis of comparison between the interference patterns corresponding to found calculated $P_{j}$ values and the patterns obtained experimentally). Whereby the possibility is envisaged for direct input of experimental information - interference patterns - from display screen interactively.

The evolvement of proposed methodology envisages, in the first place:

- determination of loading parameters of full-scale structures in the presence of inelastic deformations;

- techniques for determining nonuniform fields of residual stresses in spatial structural elements;

- techniques for determining deformation characteristics of materials. 


\section{References}

1. Gloud G.L. Optical Methods in Engineering Analyses. Cambridge: Univ. Press. 503 pp. (1998)

2. Razumovsky I.A. Interference Optical Methods of Deformable Solid Mechanics. Moscow Higher Technical University n.a. N.E. Bauman Publishing House, 237 pp. (2007)

3. Odintsev I.N. Method of Anisotropic Materials Testing under Transformable Loading Conditions. In: Machine Building and Technosphere of XXI century. Proceedings of XIV International Scientific and Technological Conference, Sevastopol, September 17-22, 2007. Donetsk: Donetsky National Technical University, 3, p. 114-118 (2007)

4. Experimental Mechanics. Ed. A. Kobayasi. Transl. from engl. In two volumes. Volume 2. M.: Mir Publishing House, 551 pp. (1990)

5. Razumovsky I.A. Determination of $K_{I}, K_{I I}$ and $K_{I I I}$ Stress Intensity Factors by Photoelastic Techniques in Uniform and Piecewise-uniform Parts and Specimens with Cracks. Factory Laboratory, 10, p. 58-64 (1988)

6. Handbook of Residual Stress and Deformation of Steel. Ed. G. Totten, M. Howes, T. Unoue. ASM International, USA, 499 pp. (2002)

7. Amosov A.A., Dubinsky Yu.A., Kopchenova N.V. Computational Methods for Engineers. Study Guide. M.: Vyisshaya Shkola Publishing House, p. 287-289 (1994)

8. Razumovsky I.A., Chernyatin A.S. Experimental and Computational Method for Loading Evaluation of Full-Scale Structures with Surface Cracks. Issues of Machine Building and Reliability of Machines, 3, 35-42 (2009) 